\title{
Complete regression of adult pineoblastoma following radiotherapy: A case report and review of the literature
}

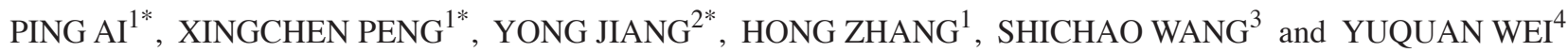 \\ ${ }^{1}$ Department of Head and Neck Oncology, State Key Laboratory of Biotherapy and Cancer Center; ${ }^{2}$ Department of Pathology; \\ ${ }^{3}$ Radiation and Physics Center, State Key Laboratory of Biotherapy and Cancer Center; \\ ${ }^{4}$ State Key Laboratory of Biotherapy and Cancer Center, West China Hospital, West China Medical School, \\ Sichuan University, Chengdu, Sichuan 610041, P.R. China
}

Received October 10, 2014; Accepted July 7, 2015

DOI: $10.3892 / 01.2015 .3574$

\begin{abstract}
Adult pineoblastomas (PBs) are rare central nervous system tumors. Little is known with regard to the clinical features and outcomes of adult PB, and optimal treatment strategies for adult $\mathrm{PB}$ remain to be determined. The current report describes a case of $\mathrm{PB}$ in a 46-year-old male, who presented with obstructive hydrocephalus due to a large pineal region mass. Considering the potential effect on quality of life, the patient underwent a partial resection. Postoperative radiotherapy, comprising prophylactic craniospinal irradiation at a dose of 34.2 Gy followed by a local 25.3-Gy 'boost' to the tumor site for a total dose of $59.5 \mathrm{~Gy}$, resulted in the complete regression of the tumor without neurological deficits. The patient has remained free of recurrence for 36 months after radiotherapy. This case highlights a minimally invasive strategy to treat a rare pineal region tumor with significant involvement of critical structures that resulted in a favorable response and an excellent neurological outcome. The radiographic and histopathological features of $\mathrm{PB}$ are also reviewed, and the various treatment options reported in the literature are discussed.
\end{abstract}

\section{Introduction}

Pineal region tumors, which account for $<1 \%$ of intracranial tumors (1), encompass a heterogeneous group of neoplasms that may be divided into four main categories: Germ cell tumors, glial cell tumors, pineal parenchymal tumors (PPTs) and other miscellaneous tumors and cysts. PPTs originate

Correspondence to: Dr Yuquan Wei, State Key Laboratory of Biotherapy and Cancer Center, West China Hospital, West China Medical School, Sichuan University, 17 Renmin South Road 3rd Section, Chengdu, Sichuan 610041, P.R. China

E-mail: yqwei222@163.com

*Contributed equally

Key words: pineoblastoma, adult, surgery, radiotherapy from cells in the pineal gland called pinealocytes, and represent only $0.3 \%$ of all primary tumors of the central nervous system (2). The current World Health Organization classification of PPTs includes well-differentiated pineocytoma (PC), PPT of intermediate differentiation and poorly differentiated pineoblastoma ( $\mathrm{PB})(3)$.

$\mathrm{PB}$ is more common in children than in adults. It has been reported that the peak incidence of $\mathrm{PB}$ occurs in the first 4 years of life, with tendency to arise in the first and second decades (4). In addition, adult cases of PB account for $<10 \%$ of published cases (5). Due to the rarity of $\mathrm{PB}$, relevant data is limited, particularly regarding PB in adults. Thus, the biology, standard management and prognosis of PB are not well understood at present.

The current report describes a case of PB occurring in a 46-year-old male who presented with obstructive hydrocephalus due to a large pineal region mass. This case highlights a minimally invasive strategy to treat a rare pineal region tumor with significant involvement of critical structures in an adult. Written informed consent was obtained from the patient.

\section{Case report}

A 46-year old male was admitted to West China Hospital of West China Medical School (Sichuan University, Chengdu, China) in June 2011 with a 3-month history of mild headache, dizziness and impaired vision. No abnormalities were observed upon physical examination and laboratory tests. An ophthalmological exam revealed no evidence of papilledema.

Computed tomography (CT) of the brain was performed, and a solid mass measuring $5 \mathrm{~cm}$ in diameter was observed in the pineal region with notable obstructive hydrocephalus. Magnetic resonance imaging (MRI) of the brain was also performed (Fig. 1), revealing a large tumor measuring $4.1 \times 5.1 \times 3.9 \mathrm{~cm}$ in the pineal region. The mass was hypointense on T1-weighted imaging and hyperintense on T2-weighted imaging, and was heterogeneously enhanced following gadolinium administration. The tumor was close to the midbrain and thalamus, and protruded forward into the posterior part of the third ventricle and backward into the cistern of the great cerebral vein. Both internal cerebral veins and the great cerebral vein were enclosed within the tumor. The mass was 
compressing the triangular region of the left lateral ventricle from its lower and medial side, and the mesencephalic aqueduct from its posterior side, and caused obstructive hydrocephalus in the supratentorial ventricles (Fig. 1A and B). MRI of the spine revealed no lesions. The differential diagnosis based on MRI included germinoma, glial tumor and ependymoma. Tests for the tumor markers $\beta$-human chorionic gonadotropin, $\alpha$-fetoprotein and carcinoembryonic antigen in the serum and cerebrospinal fluid (CSF) were negative. CSF cytology was also negative.

Due to the location of the lesion and significant involvement of critical structures, gross total resection (GTR) was not adopted and partial removal of tumor was performed. Neural guiding technology was used to locate the tumor precisely. In addition, both internal cerebral veins and the great cerebral vein were carefully identified and protected to a ensure minimally invasive procedure. Histological examination demonstrated a highly cellular tumor composed of small, round cells that were darkly stained, with hyperchromatic oval nuclei and scanty cytoplasm, and which were partially arranged in Homer-Wright rosettes (Fig. 2A). Mitosis was also observed. The majority of the tumor cells demonstrated immunoreactivity for synaptophysin (Fig. 2B), chromogranin A (Fig. 2C) and CD57 (Fig. 2D), and negativity for glial fibrillary acidic protein (Fig. 2E), S-100 (Fig. 2F) and CD99 (Fig. 2G). The Ki-67 proliferation index was $\sim 15 \%$ (Fig. 2H). Based on these findings, a pathological diagnosis of PB was determined.

Immediate postoperative MRI revealed a residual mass with gadolinium enhancement in the pineal region (Fig. 1C and D). The patient underwent subsequent external beam radiation therapy for prophylactic craniospinal irradiation (CSI) to $34.2 \mathrm{~Gy}$, followed by a local 'boost' to the tumor site for a total of $59.5 \mathrm{~Gy}$ (Fig. 3A-C). The patient was treated with $6 \mathrm{MV}$ photons in a prone position in two phases. In phase 1 , prophylactic CSI was administered to a dose of $34.2 \mathrm{~Gy}$ in 19 fractions (1.8 Gy/fraction) over 23 days using a conformal technique. In phase 2, a local 'boost' was administered to the residual gross tumor volume (GTV) at a dose of $25.3 \mathrm{~Gy}$ in 11 fractions (2.3 Gy/fraction) and to the clinical target volume (GTV plus a $1 \mathrm{~cm}$ margin) at a dose of $19.8 \mathrm{~Gy}$ in 11 fractions (1.8 Gy/fraction) over 15 days by intensity-modulated radiotherapy. During radiotherapy, treatments were tolerated extremely well by the patient, and no significant side effects were experienced.

At 2 months post radiation, MRI demonstrated that postoperative radiotherapy had resulted in complete regression of the tumor (Fig. 1E and F). At the 36-month follow-up after radiation, the patient exhibited no signs of tumor recurrence or neurological deficits (Fig. $1 \mathrm{G}$ and $\mathrm{H}$ ).

\section{Discussion}

Adult PB is rare central nervous system tumor, categorized as a supratentorial primitive neuroectodermal tumor (PNET) localized to the pineal gland, with a propensity to disseminate along the neuraxis and relapse (6). Despite certain similarities, patients with PB have different structural and immunohistochemical characteristics and such cases have poorer outcomes compared with those of infratentorial PNETs
A
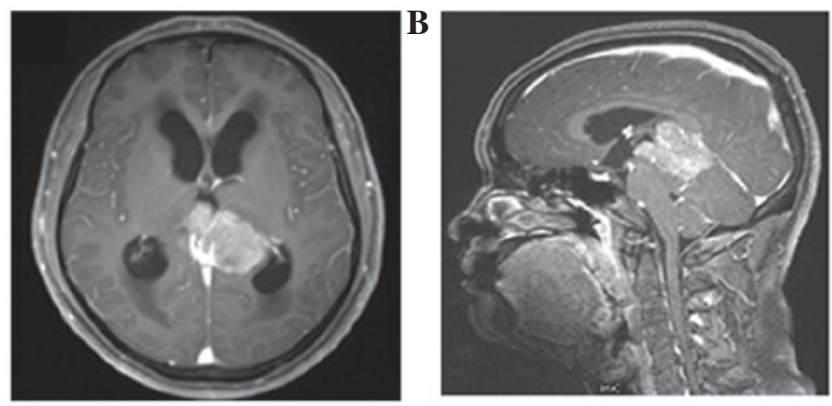

$\mathbf{C}$
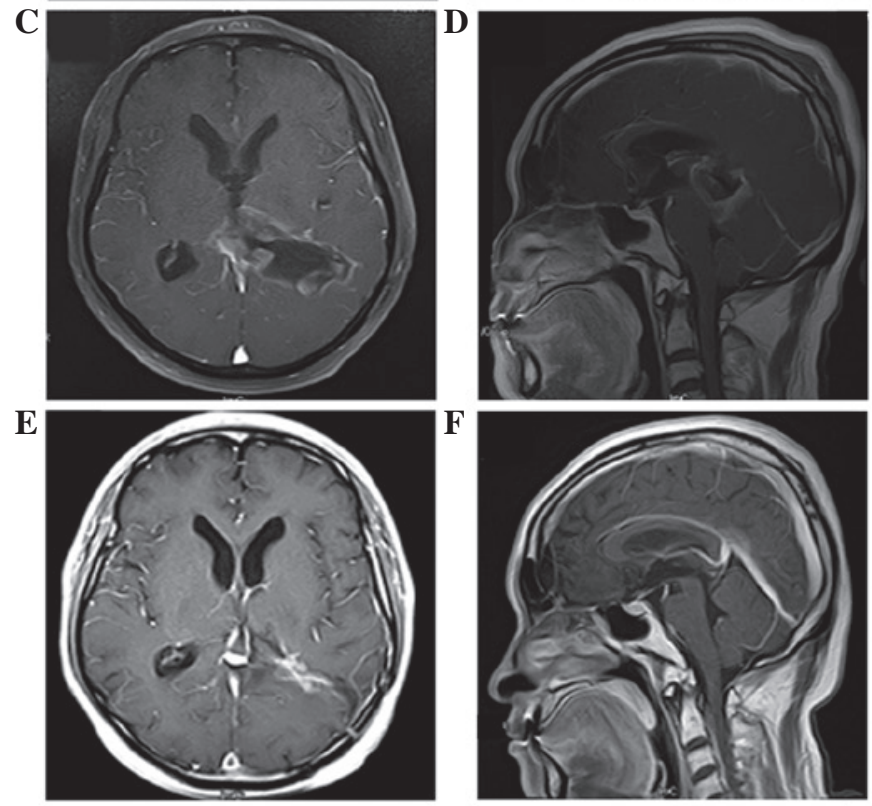

G
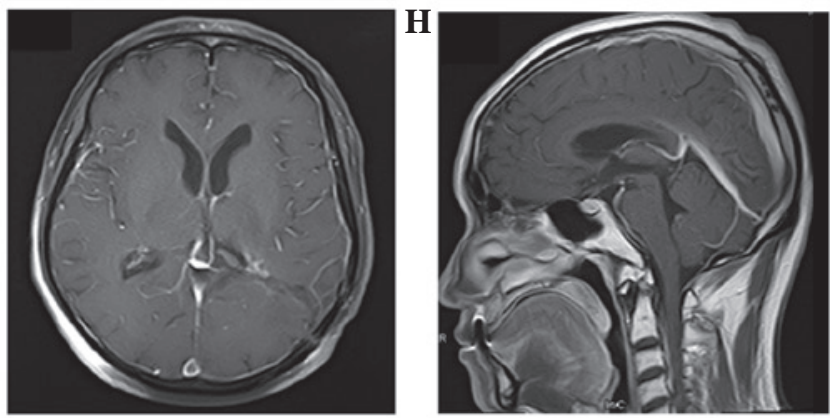

Figure 1. MRI in diagnosis and follow-up assessment of pineoblastoma. Images of the ventricle and pineal gland were captured in the axial (left column) and sagittal (right column) planes. (A and B) Initial preoperative MRI showed a massive tumor measuring $4.1 \times 5.1 \times 3.9 \mathrm{~cm}$ in the pineal region. It was heterogeneously enhanced following gadolinium administration. The tumor was close to the midbrain and thalamus, and protruded forward into the posterior part of the third ventricle and backward into the cistern of the great cerebral vein. Both internal cerebral veins and the great cerebral vein were enclosed within the tumor. It compressed the triangular region of the left lateral ventricle from its lower and medial side and mesencephalic aqueduct from its posterior side, and caused obstructive hydrocephalus in the supratentorial ventricles. (C and D) Immediate postoperative MRI. A residual mass with gadolinium enhancement in pineal region was observed. (E and F) MRI at 2 months after radiotherapy; complete regression of the tumor was observed following radiotherapy. (G and H) MRI at 36 months after irradiation; no evidence of tumor recurrence was observed. MRI, magnetic resonance imaging.

(e.g., medulloblastomas). Cases of PB in adults also have a poorer prognosis than those in children (7). Little information has been established with regard to the clinical features and outcomes of adult patients with PB. 


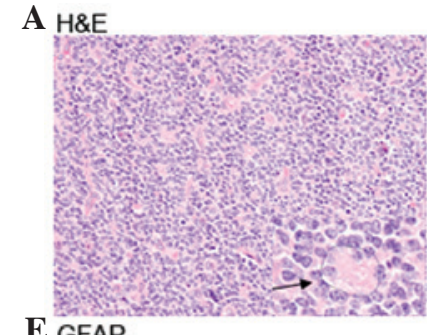

E GFAP

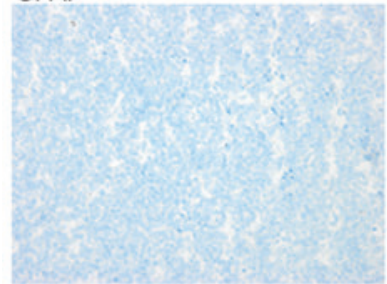

B Syn

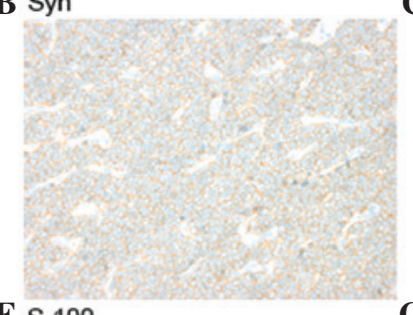

F S-100
C cgA

G CD99
D $\mathrm{CD} 57$

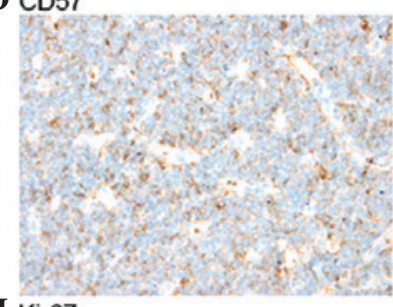

H Ki-67
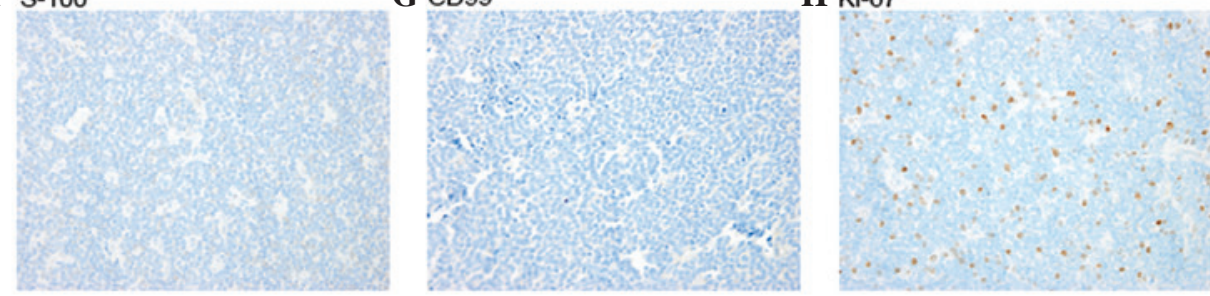

Figure 2. H\&E staining and immunohistopathology of pineoblastoma (magnification, x400). (A) H\&E staining; the highly cellular tumor was composed of small, round cells which were darkly stained with hyperchromatic oval nuclei and scanty cytoplasm and were partially arranged in Homer-Wright rosettes (arrow). (B) Positive synaptophysin immunostain. (C) Positive chromogranin A immunostain. (D) Positive CD57 immunostain. (E) Negative glial fibrillary acidic protein immunostain. (F) Negative S-100 immunostain. (G) Negative CD99 immunostain. (H) The Ki-67 proliferation index was $\sim 15 \%$. H\&E, hematoxylin and eosin.
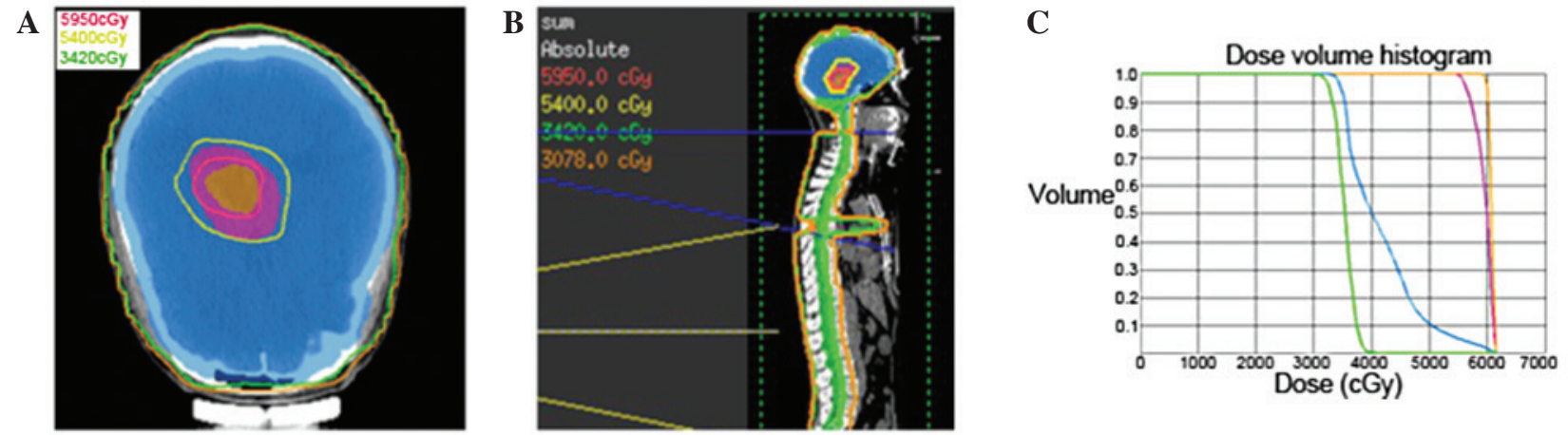

Figure 3. Target volume delineation and dose distribution. (A) Axial view of treatment plan. The PTV (orange volume) for GTV was covered by 59.5 Gy isodose line and whole brain (blue volume) was covered by 34.2 Gy isodose line. (B) Sagittal view of treatment plan. (C) Cumulative dose-volume histograms of target volumes. Orange line, PTV for GTV; pink line, PTV for clinical target volume; blue line, PTV for whole brain; green line, PTV for whole spinal cord. PTV, planning target volume; GTV, gross tumor volume.

There is no consensus on the definitive CT and MRI observations among the published cases of PB. On CT, PBs tend to be large, lobulated and heterogeneously enhanced with infrequent calcifications (8). In addition, PB often presents with a greater degree of hydrocephalus compared with PC. The tumors are typically solid and occasionally cystic, although this is more frequently observed in PC (9). On MRI, PBs are usually characterized by hypointensity or hypo to isointensity on T1-weighted images, isointensity or iso to hyperintensity on T2-weighted images, and heterogeneous enhancement in the pineal region (10). The current patient presented with a number of these radiographic characteristics.

Histologically, PB is very similar to other PNETs and has been described as 'supratentorial PNET' (6). PB is a highly cellular tumor composed of small, round, poorly differentiated cells in patternless sheets or aggregates. The cells contain hyperchromatic round or oval nuclei and scanty cytoplasm and are usually arranged in Homer-Wright rosettes, widely considered to represent abortive attempts at neuroblastic differentiation (6). Mitosis is frequently observed along with rosettes and areas of necrosis (11). In addition, the tumor cells typically demonstrate immunoreactivity for neuronal markers, such as neurofilament, synaptophysin, chromogranin A, glial fibrillary acidic protein and S-100 protein. In the current report, the majority of the tumor cells demonstrated immunoreactivity for synaptophysin and chromogranin A. The pathological diagnosis of PB primarily depends upon the location and morphology of the lesion. PB must be differentiated from other tumor types located in the pineal region, including pineocytomas, germ cell tumors and glial tumors.

The appropriate treatment strategies for $\mathrm{PB}$ have not been determined as the incidence rate of PB is extremely low, particularly in adults, and only a few described cases with limited clinical follow-up and outcome studies are available (12-14). Previous studies have reported that GTR may play a vital role in the treatment of PB $(15,16)$. However, despite significant improvements in surgical techniques and perioperative care, a high risk is associated with surgical intervention in the pineal area owing to its proximity to critical structures. It has been reported that the surgical mortality rate is $4-7 \%$ whilst the permanent morbidity rate may be up to $10 \%$ (17). 
In the present case, the tumor was not amenable to GTR due to its proximity to the midbrain and thalamus, and because both internal cerebral veins and the great cerebral vein were enclosed within the tumor. Considering quality of life, a minimally invasive strategy of partial resection followed by radiotherapy was adopted, which contributed to a favorable response with complete tumor regression and an excellent neurological outcome.

The function of postoperative therapy remains undefined in PB. A few described cases reported that radiotherapy aided in controlling the tumor and improving survival in patients with PB $(18,19)$. However, these benefits were mostly not statistically assessed due to small sample size and the lack of uniformity in radiotherapeutic strategies and doses. Lee et al (16) examined treatment factors that influenced survival in 34 adult patients who presented with PB between 1969 and 1998, and found that the median survival for patients who received $\geq 40$ Gy of cranial irradiation was three times that of patients receiving lower doses (29.8 vs. 8.1 months). However, no prospective studies have confirmed the effect of radiotherapy or optimal radiotherapeutic doses in $\mathrm{PB}$ to date.

The utilization of chemotherapy remains controversial in PB. Hinkes et al (19) demonstrated partial responses to chemotherapy in their series of six patients with PB. However, Lee et al (16) reported that chemotherapy did not confer any survival advantage among a series of 34 adult PB patients of whom 10 underwent chemotherapy (16).

In the present case, the tumor volume was great and its location was close to critical structures; therefore, partial resection followed by radiotherapy was employed in order to avoid nervous injury. Considering the tendency of PB to metastasize widely throughout the CSF pathway, prophylactic CSI was administered to a dose of 34.2 Gy. In addition, as PB is less sensitive to radiotherapy than germinoma and medulloblastoma $(20,21)$, and the residual tumor volume following surgery was large, a local 'boost' to the tumor site of $25.3 \mathrm{~Gy}$ in 2.3-Gy fractions was administered. The treatment resulted in complete tumor regression without neurological deficits.

In conclusion, $\mathrm{PB}$ is rare in adults and, although the appropriate treatment strategy for PB has yet to be determined, the current case successfully demonstrates that aggressive surgery must be avoided in patients whose tumors show significant involvement of critical structures, taking into account surgical complications, and that function-preserving resection followed by postoperative radiotherapy may be the optimal treatment strategy. However, prospective studies including a larger number of patient groups are required to demonstrate the efficacy of chemo- and radiotherapy and to determine the optimal standard treatment strategy for PB in adults.

\section{Acknowledgements}

This study was supported by grants from Wu Jieping Medical Foundation (no. 320.6750.13317), the program of Health Department in Sichuan Province (no. 130087) and the program of Science and Technology Development in Sichuan Province (no. 2014HH0063).

\section{References}

1. Al-Hussaini M, Sultan I, Abuirmileh N, Jaradat I and Qaddoumi I: Pineal gland tumors: experience from the SEER database. J Neurooncol 94: 351-358, 2009.

2. Cuccia V, Rodríguez F, Palma F and Zuccaro G: Pinealoblastomas in children. Childs Nerv Syst 22: 577-585, 2006.

3. Louis DN, Ohgaki H, Wiestler OD, Cavenee WK, Burger PC, Jouvet A, Scheithauer BW and Kleihues P: The 2007 WHO classification of tumours of the central nervous system. Acta Neuropathol 114: 97-109, 2007.

4. Nozza P, Casciana ML, Rossi A, Cama A, Milanaccio C, Raso A, Ravegnani M, Morreale G and Garrè ML: Post-chemotherapy maturation of a pineoblastoma. Acta Neuropathol 119: 651-653, 2010.

5. Hart MN and Earle KM: Primitive neuroectodermal tumors of the brain in children. Cancer 32: 890-897, 1973.

6. Herrick MK and Rubinstein LJ: The cytological differentiating potential of pineal parenchymal neoplasms (true pinealomas). A clinicopathological study of 28 tumours. Brain 102:289-320, 1979.

7. Jakacki RI, Zeltzer PM, Boyett JM, Albright AL, Allen JC, Geyer JR, Rorke LB, Stanley P, Stevens KR, Wisoff J, et al: Survival and prognostic factors following radiation and/or chemotherapy for primitive neuroectodermal tumors of the pineal region in infants and children: A report of the childrens cancer group. J Clin Oncol 13: 1377-1383, 1995.

8. Chiechi MV, Smirniotopoulos JG and Mena H: Pineal parenchymal tumors: CT and MR features. J Comput Assist Tomogr 19: 509-517, 1995.

9. Sugiyama K, Arita K, Okamura T, Yamasaki F, Kajiwara Y, Ueda $\mathrm{H}$ and Kurisu K: Detection of a pineoblastoma with large central cyst in a young child. Childs Nerv Syst 18: 157-160, 2002.

10. Fujita A, Asada M, Saitoh M, Nakamura H, Kamikawa S, Kokunai $\mathrm{T}$ and Tamaki N: Pineoblastoma showing unusual ventricular extension in a young adult-case report. Neurol Med Chir (Tokyo) 39: 612-616, 1999.

11. Schild SE, Scheithauer BW, Schomberg PJ, Hook CC, Kelly PJ, Frick L, Robinow JS and Buskirk SJ: Pineal parenchymal tumors. Clinical, pathologic and therapeutic aspects. Cancer 72: 870-880, 1993.

12. DeBoer R, Batjer H, Marymont M, Goldman S, Walker M, Gottardi-Littell N and Raizer J: Response of an adult patient with pineoblastoma to vorinostat and retinoic acid. J Neurooncol 95: 289-292, 2009.

13. Lutterbach J, Fauchon F, Schild SE Chang SM, Pagenstecher A, Volk B, Ostertag C, Momm F and Jouvet A: Malignant pineal parenchymal tumors in adult patients: patterns of care and prognostic factors. Neurosurgery 51: 44-56, 2002.

14. Gururangan S, McLaughlin C, Quinn J, Rich J, Reardon D, Halperin EC, Herndon J II, Fuchs H, George T, Provenzale J, et al: High-dose chemotherapy with autologous stem-cell rescue in children and adults with newly diagnosed pineoblastomas. J Clin Oncol 21: 2187-2191, 2003.

15. Tate M, Sughrue ME, Rutkowski MJ, Kane AJ, Aranda D, McClinton L, McClinton L, Barani IJ and Parsa AT: The long-term postsurgical prognosis of patients with pineoblastoma. Cancer 118: 173-179, 2012.

16. Lee JY, Wakabayashi T and Yoshida J: Management and survival of pineoblastoma: an analysis of 34 adults from the brain tumor registry of Japan. Neurol Med Chir (Tokyo) 45: 132-141; discussion 141-142, 2005.

17. Report of Brain Tumor Registry of Japan (1969-1993). Neurol Med Chir (Tokyo) 40 (Suppl): S1-S106, 2000.

18. Chang SM, Lillis-Hearne PK, Larson DA, Wara WM, Bollen AW and Prados MD: Pineoblastoma in adults. Neurosurgery 37: 383-390; discussion 390-391, 1995

19. Hinkes BG, von Hoff K, Deinlein F, Warmuth-Metz M, Soerensen N, Timmermann B, Mittler U, Urban C, Bode U, Pietsch T, et al: Childhood pineoblastoma: experiences from the prospective multicenter trials HIT-SKK87, HIT-SKK92 and HIT91. J Neurooncol 81: 217-223, 2007.

20. Reddy AT, Janss AJ, Phillips PC, Weiss HL and Packer RJ: Outcome for children with supratentorial primitive neuroectodermal tumors treated with surgery, radiation, and chemotherapy. Cancer 88: 2189-2193, 2000.

21. Claude L, Faure-Conter C, Frappaz D, Mottolèse C and Carrie C: Radiation therapy in pediatric pineal tumors. Neurochirurgie 61: 212-215, 2015. 